\title{
Электрическая перестройка усиления терагерцового излучения в периодической плазмонной графеновой структуре с инжекцией носителей заряда
}

\author{
(C) О.В. Полищук ${ }^{1}$, Д.В. Фатеев ${ }^{1}$, В.В. Попов ${ }^{1,2}$ \\ ${ }^{1}$ Саратовский филлиал Института радиотехники и электроники им. В.А. Котельникова Российской академии наук, \\ 410019 Саратов, Россия \\ ${ }^{2}$ Саратовский национальный исследовательский государственный университет, \\ 410012 Саратов, Россия \\ E-mail: polischuk.sfire@mail.ru, popov_slava@yahoo.co.uk
}

(Получена 25 апреля 2018 г. Принята к печати 7 мая 2018 г.)

\begin{abstract}
Теоретически исследована зависимость терагерцовой резонансной частоты плазмона в точке генерации от величины квазиэнергии Ферми на участке графена с инверсной населенностью носителей заряда и от энергии Ферми в подзатворных $p$ - и $n$-областях в периодической $p-i-n$-структуре на основе графена с инжекционной накачкой. Таким образом, показана возможность создания электрически частотно-перестраиваемых наноразмерных плазмонных графеновых усилителей и генераторов в широком терагерцовом частотном диапазоне при комнатной температуре.
\end{abstract}

DOI: 10.21883/FTP.2018.12.46752.31

\section{1. Введение}

В последнее время особое внимание уделяется плазменным колебаниям носителей заряда в графене, возбуждаемым в терагерцовом (ТГц) частотном диапазоне. Графен - двумерный монослой графита обладает представляющими большой интерес особенными электронными свойствами, вытекающими из линейного вида бесщелевого энергетического спектра носителей заряда $E= \pm V_{\mathrm{F}}|p|$ (см. вставку на рис. $\left.1, b\right)$, где $E$ и $p-$ энергия и импульс электрона (дырки) соответственно, $V_{\mathrm{F}}=10^{8} \mathrm{~cm} / \mathrm{c}-$ скорость Ферми, которая является постоянной величиной для графена, верхний и нижний знаки относятся к зоне проводимости и валентной зоне соответственно [1,2]. Графен обладает сильным плазмонным откликом на ТГц-частотах, что обусловлено как высокой плотностью, так и малой ,релятивистской“ эффективной массой свободных носителей заряда $m_{\mathrm{F}}=E_{\mathrm{F}} / V_{\mathrm{F}}^{2}$ где $E_{\mathrm{F}}$ - энергия Ферми. Экспериментальные доказательства существования плазмонов в графене приведены в работах [3,4].

Когда межзонные электронно-дырочные радиационные переходы преобладают над внутризонными потерями в инвертированном графене, действительная часть проводимости графена становится отрицательной [5], что может приводить к эмиссии ТГц-излучения из графена $[6,7]$. Теоретически предсказанное усиление плазмонов в оптически накачанном графене [6] экспериментально доказано путем наблюдения стимулированной эмиссии ТГц-фотонов с возбуждением плазмонов в графене [8]. Возникновение отрицательной высокочастотной дифференциальной проводимости графена в ТГц частотном диапазоне за счет оптической [6], инжекционной [9] и диффузионной [10] накачки было исследовано в целях создания ТГц графенового лазера.
Вследствие малой групповой скорости плазмонов и сильной локализации плазмонного поля вблизи графенового слоя, плазмонное усиление в графене с инвертированным распределением носителей заряда может на несколько порядков величины превосходить фотонное усиление [5,6,11].

Плазмоны в структурированном графене сильно связаны с электромагнитными волнами, что делает графеновые наноструктуры перспективными для создания перестраиваемых плазмонных метаматериалов в ТГц-диапазоне [12-15].

В данной работе теоретически показана возможность создания электрически перестраиваемых по частоте наноразмерных плазмонных усилителей и генераторов в ТГц частотном диапазоне на основе графена с инжекционной накачкой.

\section{2. Постановка задачи и метод решения}

Рассмотрим бесконечный лист периодически структурированного графена, расположенный в плоскости $y=0$, на поверхности подложки с вещественной диэлектрической постоянной $\varepsilon_{\mathrm{s}}$ (трехмерное схематическое изображение исследуемой структуры приведено на рис. $1, a$, один период структуры показан на рис. $1, b)$. Планарная двойная периодическая решетка представляется последовательностью проводящих металлических полосок с периодом $L$, расположенных в плоскостях $y=d, y=0$ и отделенных друг от друга слоем диэлектрика с вещественной диэлектрической постоянной $\varepsilon_{b}$. Каждая проводящая полоска двойной металлической решетки имеет нулевую толщину и бесконечна в $z$-направлении. Каждая полоска решетки в плоскости $y=d$ шириной $w_{1}=w_{3}$ отделена от соседних полосок 
$a$

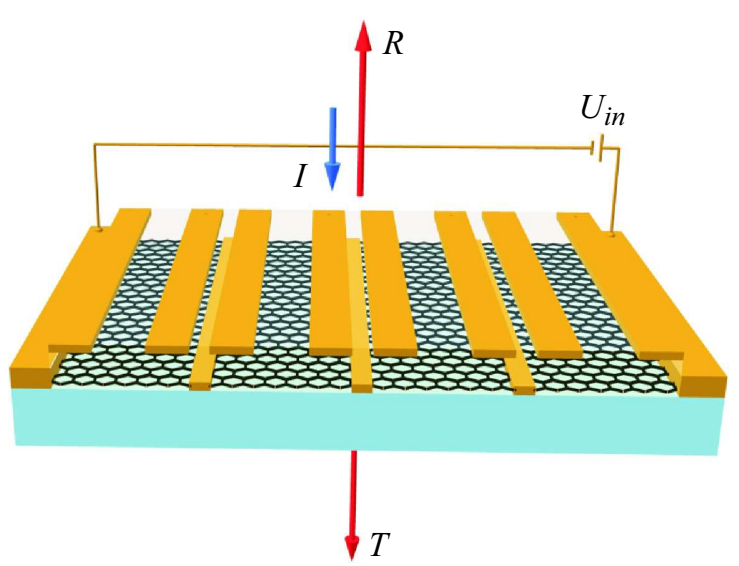

$b$

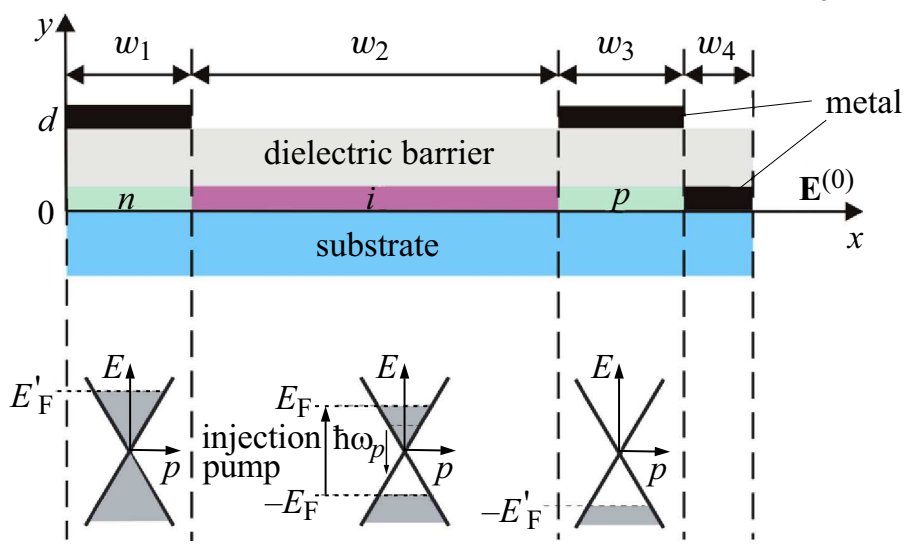

Рис. 1. $а$ - схематическое изображение нескольких периодов исследуемой структуры. Падающая $(I)$, отраженная $(R)$, и прошедшая $(T)$ волны показаны стрелками. $b-$ схематическое изображение одного периода исследуемой структуры и система координат. Внешняя ТГц-волна падает сверху в направлении нормали к плоскости структуры с поляризацией электрического поля поперек полосок металлической решетки. На вставках внизу схематически показана зонная структура электрически допированного и инвертированного графена и процесс вынужденного излучения кванта плазмона $ћ \omega_{p}$ в инжекционно накачанном графене.

разной ширины щелями. Полоски в плоскости $y=0$ шириной $w_{4}$ разделяют области структурированного графена. Двумерная проводимость металлических полосок периодической решетки (Au в нашем случае) полагалась равной $\sigma_{\mathrm{Me}}=2.5 \mathrm{OM}^{-1}$.

Окружающая среда при $y>d$ имеет диэлектрическую постоянную $\varepsilon_{\mathrm{a}}=1$ (вакуум). Подача разнополярного электрического потенциала на соседние электроды металлической решетки в плоскости $y=d$ приводит к созданию $n$ - и $p$-областей в графене, расположенных под этими электродами (рис. $1, b$ ), для которых проводимость электрически допированного графена определяется формулой $[16,17]$ :

$$
\begin{gathered}
\sigma_{\mathrm{Gr}}^{(n, p)}(\omega)=\frac{e^{2} 8 \tau k_{\mathrm{B}} T}{4 \pi \hbar^{2}(1-i \omega \tau)} \ln \left[2 \cosh \left(\frac{E_{\mathrm{F}}^{\prime}}{2 k_{\mathrm{B}} T}\right)\right] \\
+\frac{e^{2}}{4 \hbar} G\left(\frac{\hbar \omega}{2}\right)-\frac{e^{2} \omega}{i \pi} \int_{0}^{\infty} \frac{G(E)-G(\hbar \omega / 2)}{(\hbar \omega)^{2}-4 E^{2}} d E, \\
G(E)=\frac{\sinh \left(E / k_{\mathrm{B}} T\right)}{\cosh \left(E / k_{\mathrm{B}} T\right)+\cosh \left(E_{\mathrm{F}}^{\prime} / k_{\mathrm{B}} T\right)} .
\end{gathered}
$$

Здесь $E_{\mathrm{F}}^{\prime}$ - энергия Ферми в $n$ - и $p$-областях графена, температура $T$ полагается равной $300 \mathrm{~K}, e-$ - заряд электрона, $k_{\mathrm{B}}$ - постоянная Больцмана и $\hbar-$ приведенная постоянная Планка. Первое слагаемое в формуле (1) описывает отклик в форме Друде с участием внутризонных процессов с феноменологическим временем рассеяния носителей заряда в графене $\tau$. Второе слагаемое описывает межзонные переходы в электрически допированном графене. Отметим, что основной вклад в диссипацию энергии вносят внутризонные процессы. Вклад межзонных переносов мал и стремится к нулю с ростом энергии Ферми $E_{\mathrm{F}}^{\prime}$.
Инжекционная накачка электронов из $n$-области и дырок из $p$-области позволяет создать инвертированное распределение носителей заряда в межзатворной $i$-области графена, расположенной под широким просветом верхней металлической решетки. В этом случае отклик графена в инвертированной $i$-области характеризуется комплексной динамической ТГц-проводимостью [6]:

$$
\begin{aligned}
& \sigma_{\mathrm{Gr}}^{(i)}(\omega)= \frac{e^{2} 8 k_{\mathrm{B}} T \tau}{4 \pi \hbar^{2}(1-i \omega \tau)} \ln \left[1+\exp \left(\frac{E_{\mathrm{F}}}{k_{\mathrm{B}} T}\right)\right] \\
&+\frac{e^{2}}{4 \hbar} \tanh \left(\frac{\hbar \omega-2 E_{\mathrm{F}}}{4 k_{\mathrm{B}} T}\right) \\
&-\frac{e^{2} \omega}{i \pi} \int_{0}^{\infty} \frac{G\left(E, E_{\mathrm{F}}\right)-G\left(\hbar \omega / 2, E_{\mathrm{F}}\right)}{(\hbar \omega)^{2}-4 E^{2}} d E \\
& G\left(E, E^{\prime}\right)=\frac{\sinh \left(E / k_{\mathrm{B}} T\right)}{\cosh \left(E / k_{\mathrm{B}} T\right)+\cosh \left(E^{\prime} / k_{\mathrm{B}} T\right)}
\end{aligned}
$$

Квазиэнергия Ферми $E_{\mathrm{F}}$ в $i$-области графена определяется падением инжекционного напряжения на длине этой области (квазиэнергия Ферми также зависит от величины времени рассеяния, времени рекомбинации и температуры) [18]. Действительная часть проводимости (2), ответственная за диссипацию энергии, учитывает два механизма рассеяния (с участием внутризонных процессов, описываемых феноменологическим временем рассеяния носителей заряда в графене $\tau$, и межзонных переходов в инвертированном графене). Действительная часть проводимости инвертированной области графена $\sigma_{\mathrm{Gr}}^{(i)}(\omega)$ может принимать отрицательные значения при превышении пороговой величины накачки. Для осуществления инжекции носителей заряда в $i$-область необходимо, чтобы энергия Ферми в $p$ - 
и $n$-областях графена была больше, чем квазиэнергия Ферми в $i$-области графена. Инвертированная область может служить активной средой для ТГц плазмонных усилителей и лазеров. Инжекционная накачка более эффективна и проста в технологическом отношении по сравнению с оптической накачкой.

Полагаем, что плоская монохроматическая электромагнитная волна падает из вакуума нормально на плоскость исследуемой структуры. Электрическое поле падающей волны $E^{(0)} \exp \left[i k_{y}^{(0)} y-i \omega t\right]$, где $\omega-$ круговая частота, $k_{y}^{(0)}=k_{0}=\omega / c, c-$ скорость света в вакууме, поляризовано поперек полосок решетки (вдоль оси $x$ ).

Задача решалась с использованием самосогласованного электродинамического подхода, методом интегральных уравнений аналогично алгоритму, разработанному авторами в [19]. Решается полная система уравнений Максвелла с разложением искомых электрических и магнитных полей по плоским волнам. В рассматриваемой геометрии искомое индуцированное электрическое поле имеет ненулевые $x$ - и $y$-компоненты, тогда как искомое индуцированное магнитное поле имеет только ненулевую $z$-компоненту ( $p$-поляризация).

Длина волны резонансной плазмонной моды, возбуждаемой в графене падающей ТГц-волной, определяется периодом $L$ металлической решетки. Так как длина волны плазмона в графене гораздо (на 2-3 порядка) короче, чем длина электромагнитной волны на той же частоте [3,4], для возбуждения плазмонных резонансов в структуре необходимо выполнение условия $L \ll 2 \pi / k_{0}$. В этом случае только нулевые фурье-гармоники $E_{x 0}$ электрического поля соответствуют бегущим волнам, излучаемым в окружающую среду и в подложку, а все фурье-гармоники более высокого порядка характеризуют эванесцентные поля, убывающие при $y \rightarrow \pm \infty$ Следовательно, коэффициенты отражения $R$ и пропускания $T$ исследуемой структуры в области дальнего поля могут быть вычислены соответственно как $R=\left|E_{x 0}(d)\right|^{2} /\left|E^{(0)}\right|^{2}$ и $T=\left|E_{x 0}(d)\right|^{2} \sqrt{\varepsilon_{\mathrm{s}}} /\left|E^{(0)}\right|^{2}$. Коэффициент поглощения/усиления рассчитывается как $A=P_{\text {abs }} / P_{0}$, где $P_{0}-$ ТГц-мощность, падающая на единицу площади структуры, и

$$
P_{\mathrm{abs}}=\frac{1}{2 L} \int_{0}^{L} \operatorname{Re}[\sigma(\omega, x)]\left|E_{x}(x, 0)\right|^{2} d x
$$

- ТГц-мощность, поглощенная на единице площади структуры. Явный вид зависимости разрывной функции проводимости $\sigma(\omega, x)$ от координат имеет вид

$$
\begin{aligned}
& \sigma(\omega, x)= \\
& =\left\{\begin{array}{lll}
\sigma_{w 1,0}=\sigma_{\mathrm{Gr}}^{(n, p)}(\omega) \text { при } & 0<x<w_{1}, \\
\sigma_{w 2,0}=\sigma_{\mathrm{Gr}}^{(i)}(\omega) & \text { при } & w_{1}<x<w_{1}+w_{2}, \\
\sigma_{w 3,0}=\sigma_{\mathrm{Gr}}^{(n, p)}(\omega) \text { при } & w_{1}+w_{2}<x<w_{1}+w_{2}+w_{3}, \\
\sigma_{w 4,0}=\sigma_{\mathrm{Me}} & \text { при } & w_{1}+w_{2}+w_{3}<x<L
\end{array}\right.
\end{aligned}
$$

в плоскости $y=0$ и

$$
\begin{aligned}
& \sigma(\omega, x)= \\
& = \begin{cases}\sigma_{w 1, d}=\sigma_{\mathrm{Me}} & \text { при } 0<x<w_{1}, \\
0 & \text { при } w_{1}<x<w_{1}+w_{2}, \\
\sigma_{w 3, d}=\sigma_{\mathrm{Me}} & \text { при } w_{1}+w_{2}<x<w_{1}+w_{2}+w_{3}, \\
0 & \text { при } w_{1}+w_{2}+w_{3}<x<L\end{cases}
\end{aligned}
$$

в плоскости $y=d$.

Закон сохранения энергии $R+T+A=1$ выполняется как в режиме поглощения $(A>0)$, так и в режиме усиления $(A<0)$.

Расчеты выполнены для реалистичных параметров исследуемой графеновой структуры (рис. 1) с периодом 1.6 мкм при комнатной температуре $\left(\tau=10^{-12} \mathrm{c}\right)$. Период структуры состоит из четырех участков: $w_{1}=w_{3}=0.25$ мкм, $w_{2}=1$ мкм, $w_{4}=0.1$ мкм. Толщина барьерного слоя $d$ составляет 80 нм, диэлектрическая постоянная барьерного слоя $\varepsilon_{\mathrm{b}}=4$ и диэлектрической подложки $\varepsilon_{\mathrm{s}}=11.7(\mathrm{Si})$. В расчетах использовались реалистичные значения квазиэнергии Ферми $B_{\mathrm{F}}<70$ мэВ, реализуемые при инжекционной накачке графена [18].

\section{3. Результаты расчетов и их обсуждение}

Планарная двойная металлическая периодическая решетка представляет собой в данной структуре с одной стороны эффективную антенну для падающего ТГц-излучения, с другой - она образует распределенный планарный резонатор для плазмонных мод, возбуждаемых или генерируемых в графене соответственно падающим ТГц-излучением или стимулированной плазмонной эмиссией. Графен с двойной металлической периодической решеткой поддерживает плазмонные коллективные моды, когерентно колеблющиеся на всей площади структуры. Красный лепесток на рис. 2, $a$ соответствует плазмонному резонансу в режиме усиления. Усиление ТГц-волны связано со стимулированной межзонной излучательной рекомбинацией электронно-дырочных пар в $i$-области графена.

Сплошная черная линия на рис. 2 разделяет области режима усиления и режима поглощения и соответствует нулевым значениям коэффициента поглощения $(A)$. При этом усиление в активной $i$-области графена скомпенсировано поглощением в пассивных $p$ - и $n$-областях. В каждой точке рис. 2 выше сплошной черной линии усиление в активной $i$-области графена превышает поглощение в пассивных $p$ - и $n$-областях. Усиление ТГц-волны на частоте плазмонного резонанса на несколько порядков больше, чем вдали от резонансов (последнее соответствует фотонному усилению в инвертированном графене) [20]. Усиление на частоте плазмонного резонанса резко возрастает (при соответствующем резком умень- 

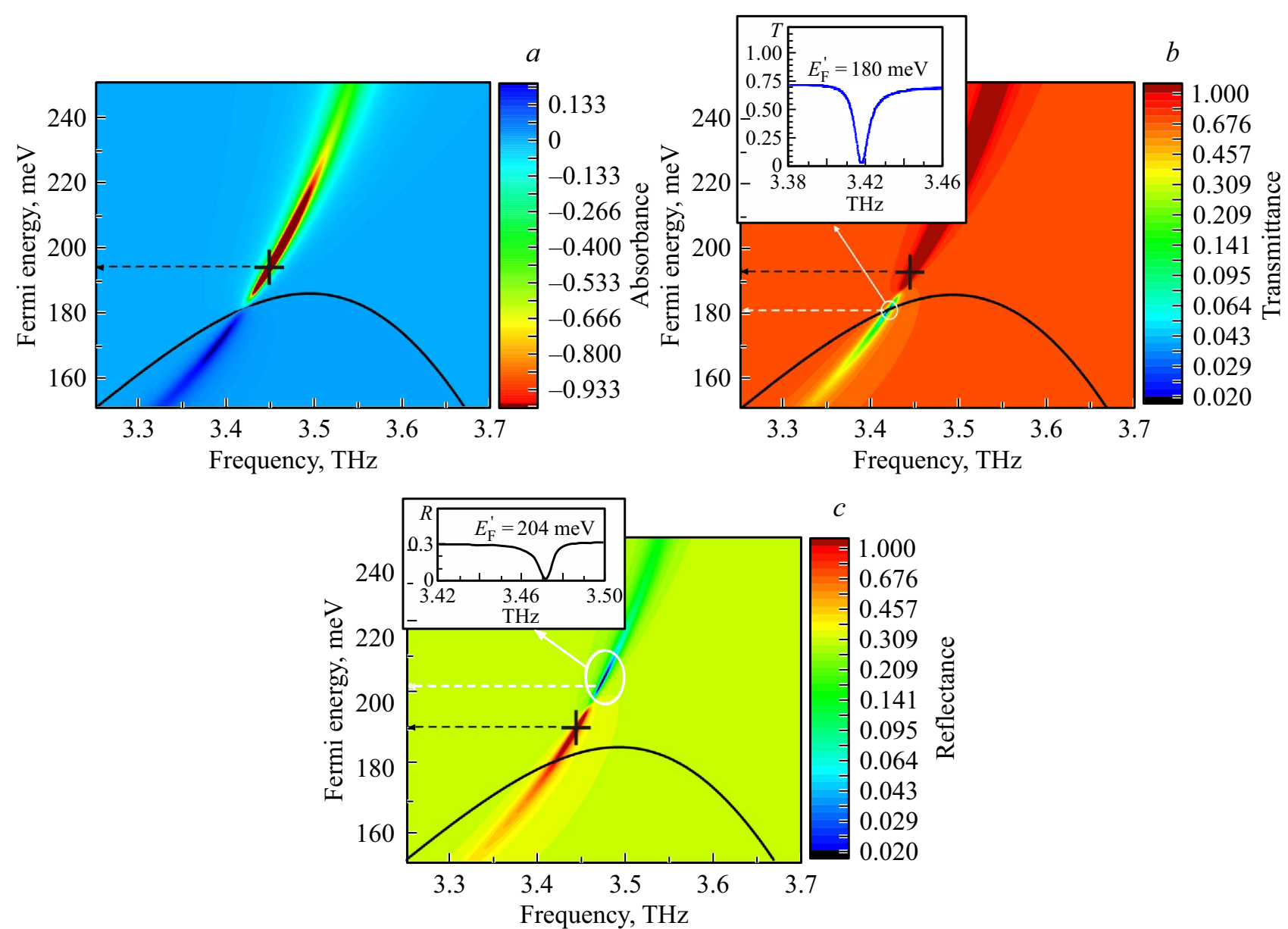

Рис. 2. $a-$ коэффициент поглощения/усиления ТГц-мощности в зависимости от энергии Ферми на подзатворных $p$ - и $n$-участках графена и частоты падающей ТГц-волны для основного плазменного резонанса. Период металлической решетки $L=1.6$ мкм, ширина области активного графена 1 мкм, электрически допированных $p$ - и $n$-участков графена -0.25 мкм. Толщина барьерного слоя $(\mathrm{Si}) d=80$ нм. Величина квазиэнергии Ферми на активном $i$-участке графена $E_{\mathrm{F}}=40$ мэВ. Крестом отмечено положение точки генерации ТГц-излучения в исследуемой структуре. $b$ - коэффициент прохождения ТГц-мощности в зависимости от энергии Ферми на подзатворных $p$ - и $n$-участках графена и частоты падающей ТГц-волны для основного плазменного резонанса. Параметры структуры те же (см. рис. 2,a). Крестом отмечено положение точки генерации ТГц-излучения в исследуемой структуре (соответствует максимуму коэффициента прохождения). Белым кружком отмечена окрестность нулевого значения коэффициента прохождения (см. вставку к рис. $2, b)$. $c$ - коэффициент отражения ТГц-мощности в зависимости от энергии Ферми на подзатворных $p$ - и $n$-участках графена и частоты падающей ТГц-волны для основного плазменного резонанса. Параметры структуры те же, что на рис. 2,a. Крестом отмечено положение точки генерации ТГц-излучения в исследуемой структуре (соответствует максимуму коэффициента отражения). Белым кружком отмечена окрестность нулевого значения коэффициента отражения (см. вставку к рис. 2,c).

шении ширины линии усиления) при определенном значении энергии Ферми $E_{\mathrm{F}}^{\prime}$ в подзатворных $p$ - и $n$-областях графена. Это соответствует режиму самовозбуждения коллективных плазмонных мод в графене с двойной металлической периодической решеткой и, как следствие, ТГц-генерации из структуры. Как было показано в работе [20], генерация наступает, когда суммарные потери энергии за счет электронного рассеяния в графене и излучения электромагнитной энергии из структуры (на активных и пассивных участках) компенсируются плазмонным усилением, что обеспечивает высокую когерентность коллективной плазмонной моды. Значение энергии Ферми $E_{\mathrm{F}}^{\prime}$, соответствующее ТГц-генерации на основном плазмонном резонансе, отмечено крестом на рис. 2, a. Как показывает поведение коэффициентов прохождения $(T)$ и отражения $(R)$ исследуемой структуры в области дальнего поля, представленных на рис. $2, b$ и $c$, поток мощности, излученной на периоде структуры, направлен в обе стороны (по ходу и против хода падающей ТГц-волны), за исключением двух особых точек. Коэффициент прохождения $T=0$ (см. вставку на рис. $2, b)$, если резонансная частота плазмона лежит на линии нулевого поглощения. Коэффициент отражения $R=0$ (см. вставку на рис. 2,c) в окрестности частот и значений уровня Ферми на пассивных подзатворных $n(p)$-участках, показанной белым кружком. 


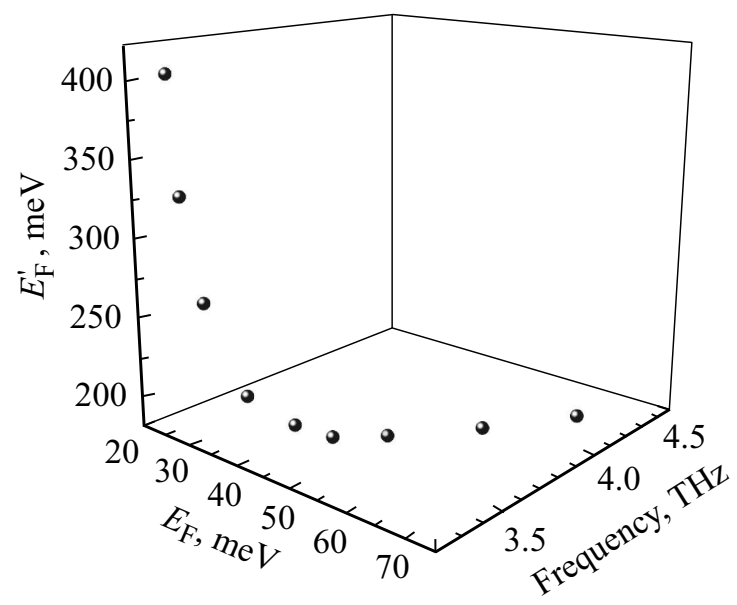

Рис. 3. Резонансная частота плазмона в точке самовозбуждения как функция величины квазиэнергии Ферми $E_{\mathrm{F}}$ в активной $i$-области графена и энергии Ферми $E_{\mathrm{F}}^{\prime}$ в подзатворных $p$ - и $n$-областях исследуемой $p-i-n$-структуры. Параметры структуры те же (см. рис. 2,a).

На рис. 3 представлена численно рассчитанная зависимость ТГц резонансной частоты плазмона в точке самовозбуждения (генерации) от величины квазиэнергии Ферми $E_{\mathrm{F}}$ на участке графена с инверсной населенностью носителей заряда (в $i$-области) и от энергии Ферми $E_{\mathrm{F}}^{\prime}$ в подзатворных $p$ - и $n$-областях.

Величина $E_{\mathrm{F}}^{\prime}$ определяется величиной разнополярного потенциала, прикладываемого к соседним полоскам металлической решетки, тогда как $E_{\mathrm{F}}$ определяется величиной тянущего напряжения, приложенного к неоднородному графену [18].

Таким образом, частота генерации может перестраиваться непрерывно в широком диапазоне ТГц-частот изменением разнополярного потенциала, прикладываемого к соседним полоскам металлической решетки, и тянущего напряжения, приложенного к неоднородному графену.

\section{4. Заключение}

Периодическая $p-i-n$-структура на основе графена с инжекцией носителей заряда поддерживает плазмонные коллективные моды, когерентно колеблющиеся на всей площади структуры. Теоретические расчеты показывают, что суммарные потери энергии за счет электронного рассеяния в графене и излучения электромагнитной энергии из структуры могут быть скомпенсированы плазмонным усилением в области инвертированного графена. Резонансная частота плазменных колебаний в графене в точке генерации может варьироваться как положением квазиуровня Ферми в активном инвертированном $i$-участке, так и положением уровня Ферми в пассивных подзатворных $n(p)$-участках. Полученные результаты показывают возможность создания компактных, электрически частотно перестраиваемых наноразмерных плазмонных усилителей и генераторов в широком ТГц частотном диапазоне на основе графена с инжекционной накачкой при комнатной температуре.

Работа выполнена при поддержке гранта РФФИ № 16-02-00814.

\section{Список литературы}

[1] K.S. Novoselov, A.K. Geim, S.V. Morozov, D. Jiang, M.I. Katsnelson, I.V. Grigorieva, S.V. Dubonos. A.A. Firsov. Nature, 438, 197 (2005).

[2] A.H. Castro Neto, F. Guinea, N.M.R. Peres, K.S. Novoselov, A.K. Geim. Rev. Mod. Phys., 81, 109 (2009).

[3] J. Chen, M. Badioli, P. Alonso-González, S. Thongrattanasiri, F. Huth, R. Hillenbrand, F.H.L. Koppens et al. Nature, 487, 77 (2012).

[4] Z. Fei, A.S. Rodin, G.O. Andreev, W. Bao, A.S. McLeod, M. Wagner. Nature, 487, 82 (2012).

[5] V. Ryzhii, M. Ryzhii, T. Otsuji. J. Appl. Phys., 101, 083114 (2007).

[6] A. Dubinov, V.Y. Aleshkin, V. Mitin, T. Otsuji, V. Ryzhii. J. Phys.: Condens. Matter, 23, 145302 (2011).

[7] V. Ryzhii, M. Ryzhii, V. Mitin, A. Satou, T. Otsuji. Jpn. J. Appl. Phys., pt 11, 50, 094001 (2011).

[8] T. Watanabe, T. Fukushima, Y. Yabe, St.A.B. Tombet, A. Satou. New J. Phys., 15, 075003 (2013).

[9] V. Ryzhii, M. Ryzhii, V. Mitin, T. Otsuji. J. Appl. Phys., 110, 094503 (2011).

[10] Ar.R. Davoyan, M.Yu. Morozov, V.V. Popov, A. Satou, T. Osuji. Appl. Phys. Lett., 103, 251102 (2013).

[11] F. Rana. IEEE Trans. Nanotechn., 7, 91 (2008).

[12] S. Thongrattanasiri, F.H.L. Koppens, F. Javier García de Abajo. Phys. Rev. Lett., 108, 047401 (2012).

[13] A.Yu. Nikitin, F. Guinea, F.J. Garcia-Vidal, L. Martin-Moreno. Phys. Rev. B, 85, 081405 (2012).

[14] F.H.L. Koppens, D.E. Chang, F.J. Garcia de Abajo. Nano Lett., 11, 3370 (2011).

[15] A. Vakil, N. Engheta. Science, 332, 1291 (2011).

[16] L.A. Falkovsky, A.A. Varlamov. Eur. Phys. J. B, 56, 281 (2007).

[17] M.S. Jang, V.W. Brar, M.C. Sherrott, J.J. Lopez, L. Kim, S. Kim, M. Choi, H.A. Atwater. Phys. Rev. B, 90, 165409 (2014).

[18] V. Ryzhii, I. Semenikhin, M. Ryzhii, D. Svintsov, V. Vyurkov, A. Satou, T. Otsuji. J. Appl. Phys., 113, 244505 (2013).

[19] О.В. Полищук, Д.В. Фатеев, В.В. Попов. ФТП, 51 (11), 1514 (2017). [O.V. Polischuk, D.V. Fateev, V.V. Popov. Semiconductors, 51 (11), 1460 (2017)].

[20] V.V. Popov, O.V. Polischuk, A.R. Davoyan, V. Ryzhii, T. Otsuji, M.S. Shur. Phys. Rev. B, 86, 195437 (2012).

Редактор А.Н. Смирнов 


\title{
Electrical tunability of terahertz amplication in a periodic plasmon graphene structure with charge carrier injection
}

O.V. Polischuk ${ }^{1}$, D.V. Fateev ${ }^{1}$, V.V. Popov ${ }^{1,2}$

${ }^{1}$ Kotelnikov Institute of

Radio Engineering and Electronics,

Russian Academy of Sciences,

410019 Saratov, Russia

2 Saratov State University, 410012 Saratov, Russia

\begin{abstract}
The dependence of the plasmon terahertz resonant frequency in the generation regime versus the value of the quasiFermi energy in the active (ungated) graphene region with the inverse population of charge carriers and the Fermi energy in the gated $p$ - and $n$-regions in a periodic $p-i-n$-structure based on graphene with injection pumping, was theoretically investigated. It has been shown that it is possible to create electrically frequency-tunable nanosized plasmon graphene amplifiers and generators in a broad terahertz frequency range at room temperature.
\end{abstract}

